\title{
Assessing Long-Term Drug Safety: Lessons (Re) Learned from Raptiva
}

\author{
Nicole M. Seminara, BA*, and Joel M. Gelfand, MS, MSCE*,+
}

\begin{abstract}
Efalizumab was approved for moderate to severe psoriasis in 2003 based on studies in approximately 2700 patients, of whom only 218 were exposed to the drug for more than 1 year. In 2009 , after more than 46,000 patients were exposed to efalizumab, the drug was withdrawn from the market after 3 confirmed and 1 suspected case of progressive multifocal leukoencephalopathy (PML) were spontaneously reported. As PML is very rare, it is extremely unlikely that the 4 reported cases were due to chance and given that PML occurs primarily in patients who are immunosuppressed, the association is likely causal. The identification of PML as a serious, but statistically rare risk of efalizumab demonstrates the strengths and weaknesses of the current drug approval and pharmacovigilance processes for fully measuring the safety of a drug. Patients and clinicians need to be aware of the relative completeness and limitations of existing safety data of a drug when selecting a treatment.
\end{abstract}

Semin Cutan Med Surg 29:16-19 @ 2010 Elsevier Inc. All rights reserved.

KEYWORDS psoriasis, pharmacovigilance, efalizumab, biologics, pharmacoepidemiology, progressive multifocal leukoencephalopathy (PML)

$\mathrm{M}$ oderate to severe psoriasis is estimated to affect about 1 million people in the United States and is associated with serious impairments in health-related quality of life, a high prevalence of psoriatic arthritis, and excess risk of myocardial infarction (MI), stroke, and mortality. ${ }^{1-8}$ Despite the serious morbidity associated with this disease, systemic therapies generally need to pass a high safety threshold to be approved for psoriasis. In 2003, efalizumab (Raptiva, Genentech, Inc., San Francisco, CA) was approved by the Food and Drug Association (FDA) for treatment of moderate to severe psoriasis based on data from its use in approximately 2700 patients. ${ }^{9}$ At the time of approval the drug appeared to be very well tolerated and the primary safety concerns that were specifically identified included the risk of serious psoriasis

*Department of Dermatology, University of Pennsylvania, Philadelphia, PA

$\uparrow$ Center for Clinical Epidemiology and Biostatistics, University of Pennsylvania, Philadelphia, PA.

This study was supported by a Graduate Research Supplement grant (RO1HL089744) from the National Heart, Lung, and Blood Institute.

Competing interests: Dr Gelfand receives grant support or is a consultant for Amgen, Centocor, Abbott, Genentech (including consulting work on a phase 4 safety study for efalizumab), Novartis, and Pfizer.

The funding sponsors had no role in the study other than providing support for Nicole Seminara's training in clinical research and epidemiology.

Address reprint requests to Joel M. Gelfand, MD MSCE, 1 convention avenue, 1471 Penn Tower, Philadelphia PA 19104. E-mail: joel.gelfand@ uphs.upenn.edu flare and the risk of thrombocytopenia, both of which were thought to be uncommon..$^{10}$ As efalizumab is immunosuppressive (it is a monoclonal antibody which binds to CDlla blocking T-cell activation and migration) there was theoretical concern for malignancies and serious infection, however the pre-approval clinical trials did not reveal a statistically significant increase in these outcomes and atypical infections were not observed. ${ }^{10}$ On February 19, 2009, after approximately 46,000 patients were exposed to efalizumab, the drug was withdrawn by regulators in the European Union and Canada when 3 confirmed cases of progressive multifocal leukoencephalopathy (PML), and 1 additional suspected, but not confirmed, case were spontaneously reported in association with efalizumab use. ${ }^{11,12}$ The drug was subsequently voluntarily withdrawn from the US market by Genentech on April 8, 2009. ${ }^{13}$ In the aftermath of these events several questions stand out: First, "How is the safety of a drug assessed after it has been approved for marketing?" and second, "How could a drug with a life-threatening side effect make it through the FDA approval process without any indication of this toxicity?"

To address these questions it is critical to understand the methods of assessing drug safety throughout the lifecycle of a drug. Initially, drugs are approved for marketing based on data from animal testing and 3 phases of clinical trials in humans. Phase 2 and 3 randomized controlled trials (RCT's) 
typically involve 500-3000 patients, and although they are the gold standard for assessing efficacy of a drug, they have numerous limitations with respect to assessing safety. ${ }^{14}$ First, RCT's usually last for a period of months, when in reality, drugs are often intended to be used for years or even decades. Therefore, adverse events that require a long duration of exposure (such as cancer, and certain types of infections) are unlikely to be detected in the short-term trials that are used to establish efficacy. Second, RCT's tend to involve highly selective patient populations; therefore, before approval the safety profile of a drug is generally not well understood in special populations, such as those with comorbidities, the elderly, children, and pregnant women. ${ }^{15,16}$ Third, RCT's occur in relatively small patient populations as described previously. As a result, these studies are generally only able to well define adverse event rates that occur in about $1 \%$ of subjects per year; rarer events such as those that occur in $0.1 \%$ of subjects per year are often not detected at all. ${ }^{15}$ Although rare side effects are unlikely to affect an individual patient and are unlikely to be observed by an individual clinician (ie, a physician would need to treat 3000 patients with a drug to be 95\% certain of observing a side effect that occurs in 1 and 1000 people), they are of public health importance given that drugs are used in very large populations of patients. For example, in 2004 alone US patients received 3.1 billion prescriptions. ${ }^{17}$

The drug approval process therefore represents a tradeoff between minimizing delays in access to new medications and our ability to fully define the safety of medications before widespread use. It is critical to recognize when a drug is approved, we only have initial information on safety. In particular, serious side effects that are delayed, rare, or more likely to occur in subpopulations (such as the elderly) are unlikely to be detected before approval. For example, the link between psoralen-ultraviolet $\mathrm{A}$ and squamous cell carcinoma was only well established after 6 years of follow up time in a cohort study following 1380 patients. ${ }^{18}$ Furthermore, the potential link between psoralen-ultraviolet A and melanoma was not observed until the cohort had been followed for approximately 20 years; a finding which still remains controversial, with most studies not finding an association. ${ }^{19,20}$ Moreover, the definitive proof that cyclo-oxygenase- 2 inhibitors increased the incidence of a relatively common event (which is still statistically rare), such as MI's, required a large randomized controlled clinical trial that followed up 2500 people for a period of 18 months. ${ }^{21}$

Due to limitations in the ability of the drug-approval process to detect events which are rare, delayed, or occur in subpopulations, the detection of new safety issues is expected after a drug has been approved. In fact, it is estimated that $51 \%$ of approved drugs have serious adverse effects not detected before approval and about $7.5 \%$ of drugs receive a black box warning after approval. ${ }^{22}$ Furthermore, during the last 4 decades, more than 130 drugs have been withdrawn from the market due to safety concerns. ${ }^{15}$ Many of these drugs had been used by hundreds of thousands of people before their withdrawal. Examples particularly relevant to dermatologists include astemizole and terfenadine (antihis- tamines that caused deaths from torsades de pointes), valdecoxib (cardiovascular risk plus the added risk of severe skin reactions), and, most recently, efalizumab.

To address the gaps in safety information that exist at the time of drug approval, pharmacovigilance, which is defined by the World Health Organization as the activities involved in the detection, assessment, understanding, and prevention of adverse effects or any other drug-related problems, is a critical aspect of assessing the risk-to-benefit ratio of therapies. ${ }^{23}$ The science of pharmacovigilance is pharmacoepidemiology. ${ }^{14}$ Pharmacoepidemiological designs used to investigate safety include hypothesis-generating descriptive studies, such as case reports, case series, and secular trend analyses, as well as hypothesis-testing analytical studies, such as case-control, cohort, and clinical trials. Most pharmacovigilance is done via spontaneous case reports submitted to the FDA (via the Medwatch program and/or the pharmaceutical companies). ${ }^{15}$ The FDA receives over 370,000 case reports of suspected adverse drug reactions annually. ${ }^{17}$ Important advantages of spontaneous reporting include that the process is relatively inexpensive, and can potentially capture data from all prescribers, drugs, patients, and dispensers. Case reports are especially useful for detecting very novel or extremely rare side effects that are generally discovered only after the drug has been used in tens of thousands of patients. For example, case reports and case series were instrumental in linking nephrogenic systemic fibrosis with gadolinium exposure, and linking toxic epidermal necrolysis with a variety of medications. ${ }^{24,25}$

The disadvantages of spontaneous reporting include the facts that adverse events are severely under-reported (only an estimated $5 \%$ of serious adverse events are reported ${ }^{26}$ ), the incidence (ie, risk) of a safety events cannot be reliably determined, a chance association is difficult to rule out, it is difficult to establish a causal relationship, and that the information may be biased because reporting is more likely to occur within the first 2 years of drug approval or if there is media attention to a particular safety issue. Therefore, case reports are best suited for signal detection to generate a hypothesis that can be further tested with analytical studies. Despite concerns over the scientific validity of spontaneous reports, they drive most postapproval safety label changes. To compound this problem, signals from case reports are not systematically tested or included in prescribing information and published case reports are rarely confirmed with controlled (ie, analytical) studies. ${ }^{27,28}$

To address the limitations of the spontaneous reporting system, the FDA has increasingly required that companies conduct additional longitudinal studies after drug approval to investigate safety signals identified in the preapproval process. Unfortunately, these postapproval studies are often not completed. In 2004, the FDA reported that only about 30\% of the 1300 postapproval studies requested had even been started and only about $15 \%$ had been completed. ${ }^{29}$ In 2007 , as a response to the public health crisis associated with cyclooxygenase-2 inhibitors, Congress passed the FDA Administration Amendments Act, which dedicated \$225 million to studying drug safety and granted the FDA 2 important new 
powers: the authority to change a drug's label after approval without negotiating with the drug's manufacturer and the ability to institute civil penalties (eg, fines) when postmarketing studies are not completed. ${ }^{30}$

Genentech itself agreed to complete 10 postmarketing safety studies for efalizumab, one of which was a cohort study following up 5000 patients treated with efalizumab for 5 years. ${ }^{31}$ Unfortunately, this study achieved less than $30 \%$ of its anticipated enrolment, and lacked a control group, severely limiting its value as a tool for detecting and interpreting safety signals. ${ }^{31}$ Prior to approval, efalizumab was studied in four phase 3 randomized, double-blind, placebo-controlled trials involving 1928 adults with moderate to severe plaque psoriasis. ${ }^{10}$ Patients were followed up for 12 weeks with an additional ongoing open-label study for 3 years. Efalizumab was generally well tolerated. In the first 12 weeks, serious infections occurred in $0.4 \%$ of efalizumab patients and $0.1 \%$ of placebo patients. ${ }^{10}$ No cases of PML or infections predominantly associated with immunosuppression were recognized, and there were no deaths. ${ }^{10,32}$ At the 24-week mark there was a decline in the rate of reported adverse events with no signs of cumulative toxicity. ${ }^{33}$ With the belief that the risk-to-benefit ratio was acceptable, efalizumab was approved in 2003 as a once weekly injection for those with moderate to severe plaque psoriasis who were candidates for systemic treatment or phototherapy. At the time of approval, 2764 people had been exposed to the drug in some capacity, but only 218 subjects had been on treatment for more than a year. ${ }^{9}$ Given the immunosuppressive nature of efalizumab, and its first in class mechanism of action, identifying safety signals related to infection and malignancy were of special concern.

By 2008 , more than 46,000 patients had been exposed to the drug and approximately 3000 patients had taken it for 2 years or longer. ${ }^{34}$ With the drug in widespread use, 3 confirmed cases of PML (and 1 highly suggestive case) were reported, beginning in 2008. PML is an extremely rare (estimated to occur at a rate of 0.15 cases per million patient years in people without human immunodeficiency virus ${ }^{35}$ ) demyelinating disorder caused by reactivation of latent JC virus that occurs almost exclusively in patients who are immunosuppressed, such as patients with acquired immunodeficiency syndrome, hematologic malignancies, and patients receiving intensive immunosuppressive therapy. ${ }^{36,37}$ Patients can present with cognitive impairment, motor dysfunction, and visual deficits. ${ }^{36}$ The disease is nearly always fatal within weeks to months of diagnosis, and there are no effective treatments. ${ }^{36,37}$ The 4 patients who developed PML (one of whom remains unconfirmed) ranged in age from 47 to $73 .{ }^{9}$ None of the patients were taking any additional immunosuppressive agents, and none were known to have any immunosuppressive conditions. All had been taking efalizumab continuously for at least 3 years. ${ }^{9}$ Based on spontaneous reports, the incidence of PML in patients treated with efalizumab is estimated to be 1 case per 15,000 person-years. ${ }^{31}$ The true incidence is likely higher as additional cases may have gone unrecognized due to the nonspecific symptoms of this disease and the well-known phenomenon of under-reporting.
Furthermore, the risk appears to be significantly higher with prolonged exposure to efalizumab. Although case reports have many limitations as described earlier, in this instance they have proved to be a very important method of identifying a safety signal that is likely causal (ie, it does not require confirmation with analytical studies) for 2 reasons: First, PML is very rare and the probability that these 4 cases occurred by chance is estimated to be less than $0.0001 \%$; and second, PML occurs almost exclusively in patients who are immunosuppressed and in the 4 reported cases associated with efalizumab there were no other factors identified that could cause immunosuppression.

In 2009, 2 months after the FDA issued a public health advisory concerning the 4 cases of PML, Genentech began a phased voluntary withdrawal of the drug from the US market and it was no longer available by June of 2009. ${ }^{13}$ Natalizumab, an immune-modulating agent that also affects T-cell migration and is used for multiple sclerosis and Crohn's disease, was also voluntarily removed from the market under the cloud of being found to cause PML. ${ }^{38}$ The risk of PML in patients taking natalizumab is about 1 in 1000 patients treated over 18 months; however, the risk increases with additional time on treatment. ${ }^{39}$ Natalizumab was later brought back to the market under a highly restrictive risk management program. Although there are an expanding number of treatment options for patients with moderate to severe psoriasis, there may still be a subgroup of patients in whom the small risk of PML is outweighed by the benefits of efalizumab, such as those with disabling disease who have failed or are intolerant of other therapies. According to a senior manager at Genentech, the company is currently undergoing discussions with the FDA to potentially establish conditions under which reintroduction may be possible. ${ }^{40}$ Risk mitigation strategies could include limiting exposure time, implementing a safety monitoring program, and only using the drug in refractory cases.

This important example of how pharmacovigilance was used to detect of the risk of PML among efalizumab-treated patients highlights the strengths and weaknesses of the current process for studying drug safety. Before approval, only 218 patients had been treated with the drug for more than 1 year. Given that the risk of PML in efalizumab patients is statistically rare and appears to increase with more prolonged drug exposure, the preapproval process was inherently unable to identify this hazard. Ultimately, identifying the risk of PML through postmarketing spontaneous reports represents a success in the current process of evaluating drug safety. Nevertheless, despite advances in the efficacy and tolerability of moderate to severe psoriasis treatments, robust long-term appropriately powered and controlled studies will be necessary to fully understand their long-term safety profile. Until we have this information, patients and clinicians need to be aware of the gaps that exist in safety data when evaluating the risk-to-benefit ratio of systemic therapies. ${ }^{41}$

\section{References}

1. Gelfand JM, Neimann AL, Shin DB, et al: Risk of myocardial infarction in patients with psoriasis. JAMA 296:1735-1741, 2006 
2. Gelfand JM, Dommasch ED, Shin DB, et al: The risk of stroke in patients with psoriasis. J Invest Dermatol 129:2411-2418, 2009

3. Gelfand JM, Feldman S, Stern R, et al: Determinants of quality of life in patients with psoriasis: a study from the US population. J Am Acad Dermatol 51:704-708, 2004

4. Gelfand JM, Gladman D, Mease P, et al: Epidemiology of psoriatic arthritis in the population of the United States. J Am Acad Dermatol 53:573, 2005

5. Gelfand JM, Troxel AB, Lewis JD, et al: The risk of mortality in patients with psoriasis: results from a population-based study. Arch Dermatol 143:1493-1499, 2007

6. Mehta NN, Azfar RS, Shin DB, et al: Patients with severe psoriasis are at increased risk of cardiovascular mortality: a cohort study using the general practice research database. Eur Heart J (in press)

7. Rapp SR, Feldman S, Exum M, et al: Psoriasis causes as much disability as other major medical diseases. J Am Acad Dermatol 4l(3 Pt. 1):401407, 1999

8. Kurd SK, Gelfand JM: The prevalence of previously diagnosed and undiagnosed psoriasis in US adults: results from NHANES 2003-2004. J Am Acad Dermatol 60:218-224, 2009

9. FDA (Feb 19, 2009). "FDA Public Health Advisory Updated Safety Information about Raptiva (efalizumab)". Press release. Retrieved 200912-05. http://www.fda.gov/Drugs/DrugSafety/PublicHealthAdvisories/ ucml10605.htm

10. Raptiva (efalizumab) [package insert]. San Francisco, CA, Genentech, 2009

11. FDA (Feb 19, 2009). "FDA Advises Public of Serious Adverse Event with Psoriasis Drug Raptiva". Press release. Retrieved 2009-12-05. http://www.fda.gov/NewsEvents/Newsroom/PressAnnouncements/ 2009/ucm 149528.htm

12. European Medicines Agency recommends suspension of the marketing authorisation of Raptiva (efalizumab). London, UK, E.M. Agency, 2009

13. FDA (Apr 8, 2009). "FDA Statement on the Voluntary Withdrawal of Raptiva from the U.S. Market". Press release. Retrieved 2009-12-05. http://www.fda.gov/NewsEvents/Newsroom/PressAnnouncements/ ucm 149561.htm

14. Strom BL. (ed): Pharmacoepidemiology, 4th ed. West Sussex, England, John Wiley \& Sons, 2005

15. Gelfand JM: Pharmacovigilance: verifying that drugs remain safe, in Wolverton SE (ed): Comprehensive Dermatologic Drug Therapy. Philadelphia, PA, Elsevier, 2004

16. Khong TK, Singer DR: Adverse drug reactions: current issues and strategies for prevention and management. Expert Opin Pharmacother 3:1289-1300, 2002

17. Okie S: Safety in numbers-monitoring risk in approved drugs. N Engl J Med 352:1173-1176, 2005

18. Stern RS, Laird N, Melski J, et al: Cutaneous squamous-cell carcinoma in patients treated with PUVA. N Engl J Med 310:1156-1161, 1984

19. Stern RS, Nichols KT, Vakeva LH: Malignant melanoma in patients treated for psoriasis with methoxsalen (psoralen) and ultraviolet A radiation (PUVA). The PUVA follow-up study. N Engl J Med 336:10411045,1997

20. Menter A, Korman N, Elmets CA, et al: Guidelines of care for the management of psoriasis and psoriatic arthritis: Section 5. Guidelines of care for the treatment of psoriasis with phototherapy and photochemotherapy. J Am Acad Dermatol 62:114-135, 2010
21. Bresalier RS, Sandler RS, Quan H, et al: Cardiovascular events associated with rofecoxib in a colorectal adenoma chemoprevention trial. N Engl J Med 352:1092-1102, 2005

22. FDA Drug Review: Postapproval risks 1976-85. Washington, DC, FDA, 1990

23. World Health Organization. The importance of pharmacovigilance: safety monitoring of medicinal products. Geneva, Switzerland, World Health Organization, 2002

24. Marckmann P, Skov L, Rossen K, et al: Nephrogenic systemic fibrosis: suspected causative role of gadodiamide used for contrast-enhanced magnetic resonance imaging. J Am Soc Nephrol 17:2359-2362, 2006

25. Stern RS, Chan HL: Usefulness of case report literature in determining drugs responsible for toxic epidermal necrolysis. J Am Acad Dermatol 21(2 Pt. 1):317-322, 1989

26. Hazell L, Shakir SA: Under-reporting of adverse drug reactions: a systematic review. Drug Saf 29:385-396, 2006

27. Loke YK, Price D, Derry S, et al: Case reports of suspected adverse drug reactions-systematic literature survey of follow-up. BMJ 332:335339, 2006

28. Avorn J: Drug warnings that can cause fits-communicating risks in a data-poor environment. N Engl J Med 359:991-994, 2008

29. FDA: Report on the performance of drug and biologics firms in conducting postmarketing commitment studies. Washington, DC, U.S. Department of Health \& Human Services, 2004

30. Schultz WB: Bolstering the FDA's drug-safety authority. N Engl J Med 357:2217-2219, 2007

31. Nijsten T, Spuls P, Naldi L: The misperception that clinical trial data reflect long-term drug safety: lessons learned from efalizumab's withdrawal. Arch Dermatol 145:1037-1039, 2009

32. Gordon KB, Papp KA, Hamilton TK, et al: Efalizumab for patients with moderate to severe plaque psoriasis: a randomized controlled trial. JAMA 290:3073-3080, 2003

33. Menter A, Gordon K, Carey W, et al: Efficacy and safety observed during 24 weeks of efalizumab therapy in patients with moderate to severe plaque psoriasis. Arch Dermatol 141:31-38, 2005

34. Barron H: Important safety information. San Francisco, CA, Genentech, 2008

35. Holman RC, Janssen RS, Buehler JW, et al: Epidemiology of progressive multifocal leukoencephalopathy in the United States: analysis of national mortality and AIDS surveillance data. Neurology 41:1733-1736, 1991

36. Korman BD, Tyler KL, Korman NJ: Progressive multifocal leukoencephalopathy, efalizumab, and immunosuppression: a cautionary tale for dermatologists. Arch Dermatol 145:937-942, 2009

37. Major EO: Progressive multifocal leukoencephalopathy in patients on immunomodulatory therapies. Annu Rev Med 61:35-47, 2010

38. FDA: Public health advisory—suspended marketing of Tysabri (natalizumab). Washington, DC, U.S. Department of Health \& Human Services, 2005

39. Yousry TA, Major EO, Ryschkewitsch C, et al: Evaluation of patients treated with natalizumab for progressive multifocal leukoencephalopathy. N Engl J Med 354:924-933, 2006

40. DeFrancesco L: RIP Raptiva? Nat Biotechnol 27:303, 2009

41. Gelfand JM: Long-term treatment for severe psoriasis: we're halfway there, with a long way to go. Arch Dermatol 143:1191-1193, 2007 